\title{
MRI MODALITIY TRANSFORMATION IN DEMON REGISTRATION
}

\author{
Dirk-Jan Kroon and Cornelis H. Slump \\ University of Twente, \\ Institute for Biomedical Technology \& Technical Medicine \\ Drienerlolaan 5, 7522 NB Enschede, The Netherlands
}

\begin{abstract}
Nonrigid local image registration plays an important role in medical imaging. In this paper we focus on demon registration which is introduced by Thirion [1], and is comparable to fluid registration. Because demon registration cannot deal with multiple MRI modalities, we introduce a MRI modality transformation which changes the representation of a $\mathrm{T} 1$ scan into a $\mathrm{T} 2$ scan using the peaks in a joint histogram. We compare the performance between demon registration with modality transformation, demon registration with gradient images and Rueckerts [2] B-spline based free form deformation method in combination with mutual information. For this test we use perfectly aligned T1 and T2 slices from the BrainWeb database [3], which we local spherically distort. In conclusion demon registration with modality transformation gives the smallest registration errors, in case of local large spherical distortions and small bias fields.
\end{abstract} ing

Index Terms - Image registration, Magnetic resonance imag-

\section{INTRODUCTION}

Quantitative analysis of Multiple Sclerosis brain lesions, e.g. analysis of progressions requires accurate segmentation. We have developed an automatic lesions segmentation system [4], which uses multiple MRI modalities of a patient FLAIR, T1,T2, MD and FA. Pixel accurate lesion segmentation is only possible with pixel accurate registration between the patient scans, thus we need an accurate multiple MRI modality registration method.

Thirion introduced a registration algorithm called 'demons algorithm' [1]. This methods is based on pixel velocities caused by edge based forces. The resulting pixel velocity / transformation field is filtered by a Gaussian kernel for global registration. It has a high registration precision [5], but is only able to register images of the same modality. A solution to allow demon registration of multiple modalities, is a representation transformation in which for example a T1 scan is changed to look like a T2 scan. In this paper, we introduce and evaluate a joint histogram based MRI representation transformation method.

\section{DEMON REGISTRATION MODEL}

\subsection{Classic Demon Registration}

The optical flow equation for finding small deformations in temporal image sequences is used as basis of the demon registration forces. For a given point $p$ in a static image $F$, let $f$ be intensity and $m$ the intensity in a moving image $M$. The estimated displacement (velocity) $\mathbf{u}$ required for point $p$ to match the corresponding point in $M$ is given by Thirion [1]

$$
\mathbf{u}=\frac{(m-f) \nabla f}{|\nabla f|^{2}+(m-f)^{2}}
$$

where $\mathbf{u}=\left(u_{x}, u_{y}\right)$ in $2 \mathrm{D}$, and $\nabla f$ is the gradient of the static image. There are two forces an internal edge based force $\nabla f$ and the external force $(m-f)$. The term $(m-f)^{2}$ is added by Thirion to make the velocity equation more stable, so to use it in image registration. Since this displacement $\mathbf{u}$ is based on local information, Gaussian smoothing of the velocity field is included as regularization. The demon equation is an local approximation, thus needs to be solved iteratively to register two images. Bro-Nielsen and Gramkow [6] demonstrated that the demon algorithm approximates the CPUexpensive viscous fluid model registration.

The original equation only uses the edges in the static image as passive internal force, He Wang et al. [7] add an equation with the image edge forces of the moving image that improves the registration convergence speed and stability.

$$
\mathbf{u}=\frac{(m-f) \nabla f}{|\nabla f|^{2}+\alpha^{2}(m-f)^{2}}+\frac{(m-f) \nabla m}{|\nabla m|^{2}+\alpha^{2}(m-f)^{2}}
$$

The normalization factor $\alpha$ is proposed by Cachier et al. [8] to adjust the force strength.

\subsection{Image Registration Model}

Vercauteren et al. [9] describe a standard registration model; with a registration energy consisting of a similarity function, a transformation error function and smoothness regularization. They use as similarity measure the squared pixel distance, and the squared gradient of the transformation field as smoothness regularization. The resulting iterative registration algorithm can be written as follows:

- Given the transformation field $S$ compute a correspondence update field $U$ by minimizing $E$,

$$
E(U)=\|F-M \circ(S+U)\|^{2}+\frac{\sigma_{i}^{2}}{\sigma_{x}^{2}}\|U\|^{2}
$$

With $F$ the static image, $M$ the moving image, transformation field $S$ describing the translation in x,y of every pixel from its original position, with $U$ the (iteration) update of $S$, ○ image transformation, and $\sigma_{i}$ and $\sigma_{x}$ a constant for intensity uncertainty (image noise) and transformation uncertainty. 
- If a fluid-like regularization is used, let $U \leftarrow K_{\text {fluid }} * U$. The convolution kernel $K_{\text {fluid }}$ is typically a Gaussian kernel.

- Update the transformation field $S \leftarrow S+U$

- A diffusion-like regularization can be included, with $S \leftarrow$ $K_{\text {diff }} * S$ (Not used in demon registration).

The update step for minimizing the energy $E(U)$ can be calculated using classic Taylor expansion. We rewrite $E$ for the pixel $p$, with $f$ the pixel intensity from static image $F$ and $m$ the intensity from the transformed image $M \circ S$, u the x,y update in translation of the pixel from $U$, and with $\nabla m$ the image gradient at pixel $p$.

$$
E(\mathbf{u})=\|f-m+\mathbf{u} \nabla m\|^{2}+\frac{\sigma_{i}^{2}}{\sigma_{x}^{2}}\|\mathbf{u}\|^{2}
$$

Then we can calculate the error gradient:

$$
\nabla E(\mathbf{u})=2(\nabla m)^{T}(f-m+\mathbf{u} \nabla m)+2 \frac{\sigma_{i}^{2}}{\sigma_{x}^{2}} \mathbf{u}
$$

Assuming that $E$ is minimum at $\nabla E(\mathbf{u})=0$, we can calculate the needed update:

$$
\mathbf{u}=\frac{f-m}{\|\nabla m\|^{2}+\frac{\sigma_{i}^{2}}{\sigma_{x}^{2}}} \nabla m
$$

We see that if we use the local estimation $\sigma_{i}(p)=|f-m|$ as the image noise and $\sigma_{x}=\frac{1}{\alpha}$ we end up with the expression of the demons algorithm in equation 1 .

\subsection{Minimizing}

Gradient descent is a basic solver for $\operatorname{argmin}_{\mathbf{x}}(E(\mathbf{x}))$, it converges not as fast as higher order minimizers. With a large number of variables as is the case with image transformation fields, it is more memory efficient.

$$
\mathbf{x} \leftarrow \mathbf{x}-\mu \frac{\nabla E}{\|\nabla E\|}, \nabla E=\left[\frac{\partial E}{\partial x_{1}}, \frac{\partial E}{\partial x_{2}}, . ., \frac{\partial E}{\partial x_{n}}\right]
$$

in which $\mu$ is the step size which is found through line search using the error equation. We can also write the extended demon Registration in gradient descent format using equation 4 as $E$ and 6 as $\nabla E$, also an active edge force can be added as in equation 2. Equation 6 is derived from $\nabla E(\mathbf{u})=0$, thus it also provides a start value $\mu$ for the line search.

\section{MODALITY TRANSFORMATION}

\subsection{Mutual Information}

Mutual information is commonly used as a similarity measure in multiple modality registration. The mutual information of moving image $M$ and static image $S$ is defined as:

$$
I(M, F)=\sum_{M, F} p(m, f) \log \left(\frac{p(m, f)}{p(m) p(f)}\right)
$$

In this equation $p(m)$ and $p(f)$ are the probabilities of the gray values in resp. image $M$ and $F, p(m, s)$ is the joint probability of the images gray values which can be derived from the joint histogram. Mutual information is global and gives only one similarity value for the whole image area, which is a disadvantage when using finite difference methods for local registration.
The idea behind mutual information registration is that every image has certain uniform intensity regions separated by edges, these regions correspond with regions in another image but with different intensity and texture. In an iterative registration process, corresponding regions will overlap more increasing the peaks in the joint histogram.

\subsection{Proposed method}

We propose to use the joint histogram peaks to transform one image representation in to the other, allowing fast intensity based local image registration such as demon registration.

The joint histogram $H(I, J)$ of image $I$ and $J$ can be written as 9, looping through all pixel locations

$$
H(\lfloor I(\mathbf{x}) N\rfloor,\lfloor J(\mathbf{x}) N\rfloor)=H(\lfloor I(\mathbf{x}) N\rfloor,\lfloor J(\mathbf{x}) N\rfloor)+1
$$

With $N$ the number of bins and with $I, J \in[0,1]$ and $\mathbf{x}$ is the pixel location.

We transform the image $I$ into $I_{T}$ with the same representation as $J$. This is done by finding for every pixel the gray value in image $J$ which overlaps most often with the pixel gray value in image $I$.

$$
I_{T}(\mathbf{x})=\operatorname{argmax}_{j}(H(\lfloor I(\mathbf{x}) N\rfloor,\lfloor j N\rfloor))
$$

In medical images two regions can have the same gray value in one modality, but in another both regions can have totally different gray values. Also medical images suffer from slowly varying intensity non uniformities called the bias field in MRI . This implies that we have to use a more local modality transformation. We solve this problem by calculating a separate local mutual information histogram for every pixel by using Gaussian windows.

\subsection{Combined with Demon Registration}

When we transform an image from one MRI representation to another, the transformation is poorly defined on edges of the image, and the new image can contain some false edges. Thus a modality transformed image is not very useful to serve as edges forces. Thus our final demon registration algorithm with representation transformation ${ }^{1}$ is:

$$
\begin{array}{r}
E=\frac{1}{2}\left\|F_{T}-M \circ(S+U)\right\|^{2} \\
+\frac{1}{2}\left\|F-M_{T} \circ(S+U)\right\|^{2}+\frac{\sigma_{i}^{2}}{\sigma_{x}^{2}}\|U\|^{2}
\end{array}
$$

$$
\begin{aligned}
\nabla E & =\left(M_{T} \circ S-F\right)\left(\frac{\nabla F}{|\nabla F|^{2}+\alpha^{2}\left(M_{T} \circ S-F\right)^{2}}\right) \\
& +\left(M \circ S-F_{T}\right)\left(\frac{\nabla M}{|\nabla M|^{2}+\alpha^{2}\left(M \circ S-F_{T}\right)^{2}}\right)
\end{aligned}
$$

With $E$ the registration error, $M_{T}$ and $F_{T}$ the modality transformed static and moving image, $S$ the transformation field, $U$ the update of the transformation field (used by the line search).

To avoid local minima and to speed up registration, a scale space approach is used. We first resize the original images to $8 \times 8$ pixels and register these small images. Next we resize the found transformation fields and original images to $16 \times 16$, and so on, until the original resolution is reached.

\footnotetext{
${ }^{1}$ Matlab implementation available on MathWorks.com 'File Exchange'
} 


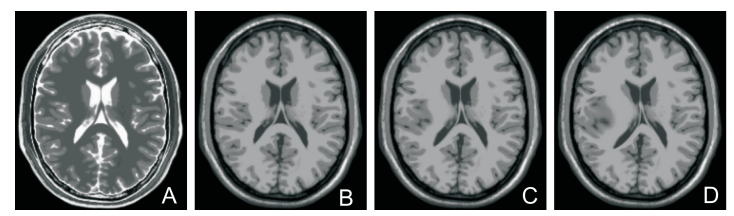

Fig. 1. Figure A and B shows a T2 and T1 slice without noise or bias field. Figure $\mathrm{C}$ and $\mathrm{D}$ shows the local spherize transformed T1 slice with a $\gamma$ value of 30 and 60 degrees

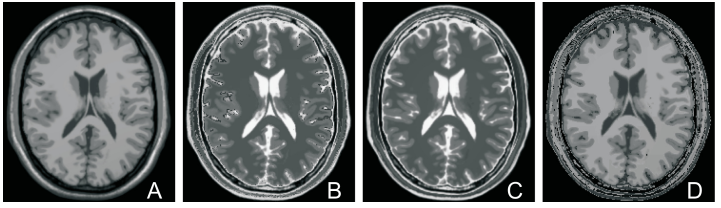

Fig. 2. Figure $\mathrm{A}$ and $\mathrm{C}$ shows a $\mathrm{T} 1$ and $\mathrm{T} 2$ slice without noise or bias field, which are modality transformed into image, B and D, respectively.

\section{RESULTS}

\subsection{Setup}

To test the performance of the demon registration algorithm we need perfect aligned ground truth data from multiple modalities. For this reason we use the BrainWeb MRI Simulated Normal Brain Database [3]. This database can provide $\mathrm{T} 1$ and $\mathrm{T} 2$ images with several noise and bias configurations. The noise in the simulated images has Rayleigh statistics in the background and Rician statistics in the signal regions. The 'percent noise' number represents the percent ratio of the standard deviation of the white Gaussian noise versus the signal for a reference tissue. Bias fields are varying fields estimated from real MRI scans; for a $20 \%$ level, the multiplicative biasfield has a range of values of 0.90 to 1.10 over the brain area [10].

Because demon registration is developed for local non-rigid transformation, we test the our algorithm using a spherical distortion (spherize filter) on an part of a $\mathrm{T} 1$ brain slice, location center slice $\mathrm{z}$-plane (90), x,y coordinate 115,60 radius 34 . The spherical distortion is described by [11]:

$$
\begin{gathered}
R=R_{0} /\left(\sqrt{2} \sin \left(\frac{\gamma}{2}\right)\right) \\
X_{c}=\frac{1}{2}\left(1+\cot \left(\frac{\gamma}{2}\right)\right) R_{0}, Y_{c}=\frac{1}{2}\left(1-\cot \left(\frac{\gamma}{2}\right)\right) R_{0} \\
{\left[\begin{array}{l}
\dot{x} \\
\dot{y}
\end{array}\right]=\frac{Y_{c}+\sqrt{R^{2}-\left(\sqrt{x^{2}+y^{2}}-X_{c}\right)^{2}}}{\sqrt{x^{2}+y^{2}}}\left[\begin{array}{l}
x \\
y
\end{array}\right]}
\end{gathered}
$$

With $R_{0}$ the $2 \mathrm{D}$ radius of the distortion, $R$ the radius of the virtual 3D sphere, $\gamma$ the amount of distortion range 0 to 90 degrees, pixel coordinates $x, y$ with $x, y$ the spherical transformed coordinates

An example of the transformed images are shown in figure 1 . Before the demon registration can be done both the $\mathrm{T} 1$ and $\mathrm{T} 2$ are transformed to their opposite MRI representations, using local joint histogram peaks between the T1 and T2 image, see figure 2 .

The parameters in the demon registration are chosen to suppress noise but still allow local transformations, $\sigma$ of the transformation Gaussian smoothing is $8, \alpha$ is chosen 2.5. The Gaussian window

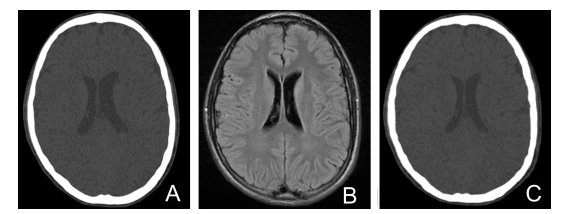

Fig. 3. A CT slice (A) is registered with demon registration on a T1 (B) slice of the same patient,figure $\mathrm{C}$ shows the result.

used for modality transformation is chosen $100 \times 100$ with $\sigma=33$, and after registration, a Gaussian window is used for modality transformation with size $70 \times 70$ and $\sigma=23$ followed by a second registration pass.

\subsection{Methods used as comparison}

We compare the registration performance with the free form deformation (FFD) registration method grid existing of 1D B-splines which is introduced by Rueckert et al. [2]. The control points of the grid are moved to transform an image, and a similarity measure between a target image and transformed image is used to determine if the registration improves. We have implemented the algorithm including multi-scales refinement, and for fast and stable mutual information registration, we calculate the mutual information measure separately for each control point, from his neighborhood.

Edges of the regions in $\mathrm{T} 1$ and $\mathrm{T} 2$ can be registered onto each other, for comparison to our representation transformation method. In [12] the normalized gradient field is used. We have tested the normalized gradient field and a canny edge detector transforming both T1 an T2 to the same "edge representation'. These approaches give large registration errors, because some region edges are detected in $\mathrm{T} 1$ but not in T2. Finally we decided to high pass filter the images and normalize the images with $|I| /(|I|+\beta)$, with constant $\beta=0.1$ this gave more reliable results.

\subsection{Simulations}

The first simulation is by spherical transforming a part of a bias and noise free $\mathrm{T} 1$ slice as in figure 1 . We show the effect of the amount of distortion between a $\mathrm{T} 1$ an $\mathrm{T} 2$ slice versus registration result. The mean transformation error is calculated on the area of the spherical distortion $70 \times 70$, and is the distance in position between the correct pixel location and location after registration. The transformation error after registration is shown for the B-spline, demon and gradient registration, see figure 4. Modality transform with demon registration clearly outperforms the other registration methods. The spherical distortion has non smooth transformation edges, thus the Rueckert B-spline registration which produces curvature smooth transformation fields performs less for a spherical distortion of more than 30 degrees. The Gradient Registration has an error due to edges which are not present simultaneously in both modalities.

The second simulation is to test the influence from a bias field on the registration. A bias field will broaden the histogram peaks used for detection, thus tissues cannot be classified by one gray value. This problem is partly solved in our representation transformation method by using a local Gaussian window for the joint histogram. The results can be found in figure 5 . 


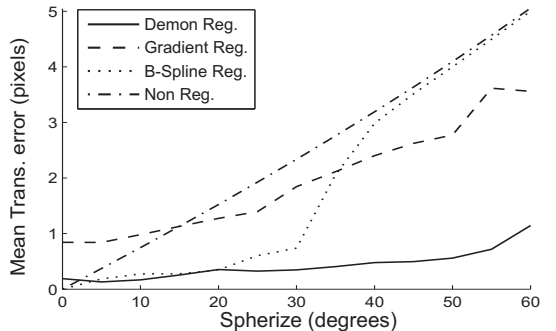

Fig. 4. Registration performance with increasing distortion. A spherical transformed $\mathrm{T} 1$ slice is registered on a $\mathrm{T} 2$ slice, with modality transformed demon registration, gradient images registration and B-spline registration.

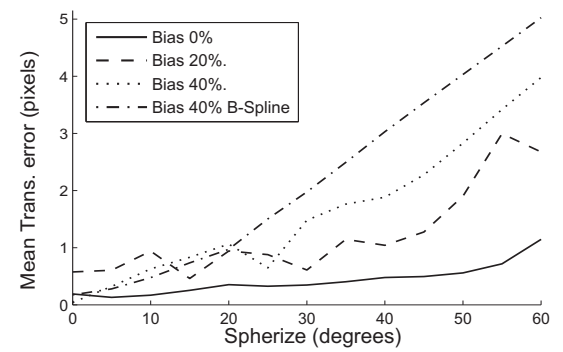

Fig. 5. Bias field registration performance. With spherical transformed $\mathrm{T} 1$ slice registered on $\mathrm{T} 2$ slice

The final simulation is to test the influence of noise on the registration result, see figure 6 . With increasing noise the transformation error increases slightly. From zero to one percent noise the registration error becomes better, this is due to local minima during registration. A simulated annealing optimizer is most likely better than the current gradient decent.

\section{CONCLUSIONS}

Modality transformation using the intensity peaks in a joint histogram seems to work very well for deformed MRI images, and probably also with CT see figure 3. The bias field has small effect on the registration error with small deformations, but with large deformations doubling the bias fields will also double the transformation error. Gradient / Edge based registration suffers from the fact that region edges do not show in all modalities, which results in incorrect transformations during registration with aligned image data. This problem can be solved by using a wider Gaussian to smooth the registration velocity field, but in that case registration is no longer local. Rueckerts B-spline registration is not capable to deal with large spherical deformations, probably because the b-spline grid can only represent really smooth transformations. In conclusion demon registration with image transformation gives the best results while dealing with large spherical distortions and good T1 and T2 MRI images. The registration method used can probably be improved by new modality transformations during the demon registration iterations and using an simulated annealing optimizer.

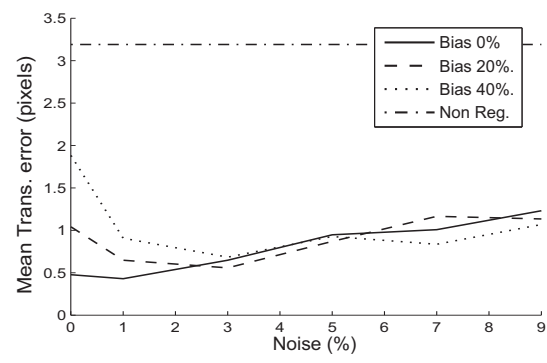

Fig. 6. Noise registration performance. Spherical transformed T1 slice registered on $\mathrm{T} 2$ slice, both with bias fields.

\section{REFERENCES}

[1] J.P. Thirion, "Image matching as a diffusion process: an analogy with maxwell's demons," Medical Image Analysis, pp. 243-260, September 1998.

[2] D. Rueckert, L. Sonoda, C Hayes, D.L.G. Hill, M Leach, and D.J. Hawkes, "Non-rigid registration using free-form deformations: Application to breast MR images," IEEE Transactions on Medical Imaging, vol. 18, no. 8, pp. 712-721, 1999.

[3] C.A. Cocosco, V. Kollokian, R.K.-S. Kwan, G.B. Pike, and A.C. Evans, "Brainweb: Online interface to a 3D MRI simulated brain database," NeuroImage, vol. 5, pp. 425, 1997.

[4] D. Kroon, E. van Oort, and K. Slump, "Multiple sclerosis detection in multispectral magnetic resonance images with principal components analysis," IJ - 2008 MICCAI Workshop - MS Lesion Segmentation, 2008.

[5] F.S. Castro, C. Pollo, O. Cuisenaire, J. Villemure, and J. Thiran, "Validation of Experts Versus Atlas-Based and Automatic Registration Methods for Subthalamic Nucleus Targeting on MRI," International Journal of Computer Assisted Radiology and Surgery, vol. 1, no. 1, pp. 5-12, 2006.

[6] M. Bro-Nielsen and C. Gramkow, "Fast fluid registration of medical images," 1996, pp. 267-276, Springer-Verlag.

[7] H. Wang, L. Dong, J. O'Daniel, R. Mohan, A.S. Garden, K.K. Ang, D.A Kuban, J.Y. Bonnen, M. Chang, and R. Cheung, "Validation of an accelerated 'demons' algorithm for deformable image registration in radiation therapy," Physics in Medicine and Biology, vol. 50, no. 12, pp. 28872905, 2005.

[8] P. Cachier, X. Pennec, and N. Ayache, "Fast non-rigid matching by gradient descent: study and improvement of the demons algorithm," 1999.

[9] T. Vercauteren, X. Pennec, A. Perchant, and N. Ayache, "Nonparametric diffeomorphic image registration with the demons algorithm," Medical Image Computing and Computer-Assisted Intervention MICCAI 2007, pp. 319-326, 2007.

[10] R.K.S. Kwan, A.C. Evans, and G.B. Pike, "MRI simulationbased evaluation of image-processing and classification methods," IEEE Transactions on Medical Imaging, vol. 18, no. 11, pp. 1085-1097, 1999.

[11] F. Chen and K. Arunachalam, "Geometric transformation pinched hallway and its restoration," 2001.

[12] E. Haber and J. Modersitzki, Bildverarbeitung für die Medizin 2005, vol. 5, pp. 350-354, Springer-Verlag, 2005. 